\title{
Use of Finite Difference Method to Study a Model System of Nitrification at Low Substrate Concentrations
}

\author{
By J. I. PROSSER AND T. R. G. GRAY \\ Hartley Botanical Laboratories, University of Liverpool, P.O. Box I47, \\ Liverpool L69 $3 B X$
}

(Received 10 February 1977; revised 6 May 1977)

A mathematical model of nitrification in a column is developed in which time $(t)$ and distance are considered independently, using a finite difference approach incorporating the following equations for removal of substrate $(S)$ and growth of biomass $(m)$ :

$$
\begin{aligned}
\frac{\mathrm{d} S}{\mathrm{~d} t} & =\frac{\mu S m}{K_{\mathrm{s}}+S}-D S \\
\frac{\mathrm{d} m}{\mathrm{~d} t} & =\frac{\mu S m}{K_{\mathrm{s}}+S}-k m^{2}
\end{aligned}
$$

where $\mu$ is the maximum specific growth rate, $K_{\mathrm{s}}$ the saturation constant for growth, $k$ a growth limiting factor and $D$ the dilution rate. This model gives new qualitative and quantitative predictions and in particular predicts overshoots and undershoots in conversion product concentrations following decreases and increases in dilution rate. The model was tested using an experimental system consisting of a column of glass beads, inoculated with Nitrobacter, through which was passed a constant flow of medium containing I p.p.m. $\mathrm{N}$ as $\mathrm{NO}_{2}^{-}$; effluent was analysed for nitrite and nitrate.

Short undershoots, lasting several hours, were due to reactivation of bacteria following nutrient starvation. Expected overshoots and undershoots lasting several days were also observed. However, these were not asymmetrical, as predicted, since washout of cells as a limiting growth factor and delay of adjustment to growth rate to different substrate concentrations were not considered.

\section{INTRODUCTION}

Most models describing nitrification in the soil consider ammonium and nitrite conversion to follow first-order rate kinetics with bacterial growth kinetics following the logistic equation. The latter assumption may be reasonable where substrate is present at non-growth limiting concentrations (Prosser \& Gray, 1977). However, this is rarely true in soil, and even where, for example, ammonium is added at high concentration as fertilizer, nitrite will initially be low and this must be considered when describing Nitrobacter growth.

Saunders \& Bazin (I973) considered a column of constant cross-sectional area packed with particular material and inoculated with Nitrosomonas and Nitrobacter, through which a solution containing ammonium is passed at constant rate. As ammonium passes down the column it is converted to nitrite with a concomitant increase in the numbers of Nitrosomonas. If the incoming ammonium concentration is high, then even at the base of the column Nitrosomonas growth may not be limited. However, at the top of the column there is no nitrite. This is formed by Nitrosomonas as the liquid flows down the column and is then converted to nitrate by Nitrobacter. Thus, the nitrite concentration will vary within the 
column and at the top at least will be growth-limiting for Nitrobacter. As pointed out by Saunders \& Bazin (1973), both time and position must be considered as independent variables for correct description of this system.

They overcame this problem by use of partial differential equations in which first-order rate kinetics were assumed and two different expressions for bacterial growth were considered. The first of these involved the logistic equation and an analytical solution was obtained. The second considered substrate limitation of the form:

$$
\frac{\mathrm{I}}{m} \cdot \frac{\partial m}{\partial t}=\frac{\mu S}{K_{\mathrm{s}}+S}
$$

where $m$ is the biomass, $S$ the substrate concentration, $t$ time, $K_{\mathrm{s}}$ the saturation constant and $\mu$ the maximum specific growth rate.

This model cannot be used to predict the effect of transient conditions such as change in flow rate and an analytical solution required several simplifying assumptions thereby decreasing the range of substrate concentrations over which it was valid.

An alternative to the use of partial differential equations is a finite difference approach in which the column is considered to be split into a number of theoretical compartments. Such a model is described here along with experiments to test the predictions obtained.

\section{THE MODEL}

The model considers a column of constant cross-sectional area containing inert particulate matter, e.g. glass beads, inoculated (initially uniformly) with nitrifying bacteria through which is passed medium containing ammonium or nitrite. For simplicity, conversion of nitrite to nitrate will be considered. The column is considered as a number of theoretical compartments of equal size, this size being determined by the time taken for the solvent front to pass through the compartments so that any solvent element is in each compartment for an equal amount of time, $t_{\mathrm{L}}$. The actual number and length of individual compartments consequently depends on flow rate, faster rates giving fewer and larger compartments. The number of compartments $(n)$ is given by the equation:

$$
n=V \mid f t_{\mathbf{L}}
$$

where $V$ is the volume of liquid medium in the column (void volume) and $f$ the flow rate within the column.

Lees \& Quastel (1946) found that nitrification took place at the soil surface and that activity in the soil solution was negligible. Nitrobacter is therefore assumed to be attached to the particles but in all other respects each compartment is treated as a normal continuous culture system with input and output of all but Nitrobacter.

Changes in nitrite and Nitrobacter are then computed for the time interval $t_{\mathrm{L}}$ with the input nitrite concentration for compartment 2 being equal to the output from compartment I from the previous time interval, and so on down the column. The input nitrite concentration for compartment $\mathrm{I}$ is that of the inflowing medium and the output nitrite and nitrate concentrations from the last compartment are those of the effluent. Because of the above assumption regarding attachment of bacteria, no transfer of biomass between compartments is incorporated in the model.

At the end of each time interval $t_{\mathrm{L}}$, the non-fixed components, i.e. nitrite and nitrate concentrations, are moved down one compartment while the fixed components are held in the compartments. The dilution rate $(D)$ for the whole column is given by:

$$
D=f / V
$$

The volume of each compartment $(v)$ is given by:

$$
v=V / n
$$


But $n=V \mid f t_{\mathrm{L}}$

Therefore $v=f t_{\mathrm{L}}$

and the dilution rate for each compartment $\left(D_{c}\right)$ is given by:

$$
D_{\mathrm{c}}=f \mid f t_{\mathrm{L}}=\mathrm{I} / t_{\mathrm{L}}
$$

Thus the dilution rate for each compartment is the reciprocal of the residence time in that compartment and this value may be used when computing component changes over a time interval of unity. If, as described above, this time interval is also equal to $t_{\mathrm{L}}$ then $D_{\mathrm{c}}$ must be divided by $\mathrm{I} / t_{\mathrm{L}}$ giving a value of $D_{\mathrm{c}}=\mathrm{I}$.

If the flow rate is changed from one value to another, then the number of compartments will also change if $t_{\mathrm{L}}$ is to be kept constant, and values of nitrite and nitrate concentrations and biomass must be provided for the new compartments. This is done by considering two columns, $\mathrm{I}$ and 2, at flow rates $f_{1}$ and $f_{2}$ with $n_{1}$ and $n_{2}$ compartments respectively, and an intermediate column, 3 , with $n_{3}=n_{1} \times n_{2}$ compartments. For a change of flow rate from $f_{1}$ to $f_{2}$, values of, for example, nitrite concentration are calculated for column 3 using the equation:

$$
S_{3, i}=S_{1, j} \quad \text { for } i=(j-\mathrm{I}) n_{1}+\mathrm{I} \text { to } j n_{1}
$$

where $S_{3, i}$ is the nitrite concentration, nitrate concentration or biomass in compartment $i$ in column 3 , and $S_{1, j}$ is the nitrite concentration, nitrate concentration or biomass in compartment $j$ in column $\mathrm{I}$.

For a change of flow rate, values of $S_{1}$ are first transferred into column 3 and values of $S_{2}$, i.e. concentrations at flow rate $f_{2}$, are given by the equation:

$$
S_{2, i}=\frac{{ }_{(i-1) n_{1}+1}^{i n_{1}} \sum S_{3, j}}{n_{1}}
$$

This method permits conversion of either batch or continuous flow models into models for the column system, and distribution of components with time and distance can be obtained easily and simultaneously. While the method represents an approximate approach, this is not thought to be a serious disadvantage as the errors involved may be reduced by increasing the number of compartments; of the other models, only the simplest provide complete analytical solutions.

The approach of Saunders \& Bazin (1973) is more fundamentally correct than the finite difference method and does provide an analytical solution for the case of logistic growth. For substrate-limited growth, however, numerical methods are required and a solution is only obtained using simplifying assumptions unnecessary in the above treatment. The finite difference approach also allows prediction of behaviour following a change in flow rate which is not possible for Saunders \& Bazin's model.

Equations used to represent nitrification in each compartment were based on those of Bazin \& Saunders (I973) which, for oxidation of nitrite to nitrate, have the form:

$$
\begin{aligned}
\frac{\mathrm{d} S_{1}}{\mathrm{~d} t} & =D\left(S^{0}-S_{1}\right)-\frac{\mu S_{1} m}{K_{\mathrm{s}}+S_{1}} \\
\frac{\mathrm{d} m}{\mathrm{~d} t} & =\frac{\mu S_{1} m}{K_{\mathrm{s}}+S_{1}}-k_{1} m^{2} \\
\frac{\mathrm{d} S_{2}}{\mathrm{~d} t} & =k_{2} m-D S_{2}
\end{aligned}
$$

where $D$ is the dilution rate, $S_{1}$ the nitrite concentration, $S^{0}$ the nitrite concentration in the inflowing medium, $\mu$ the maximum specific growth rate of Nitrobacter, $m$ the mass of Nitrobacter, $K_{\mathrm{s}}$ the saturation constant, $S_{2}$ the nitrate concentration, and $k_{1}$ and $k_{2}$ are constants. 


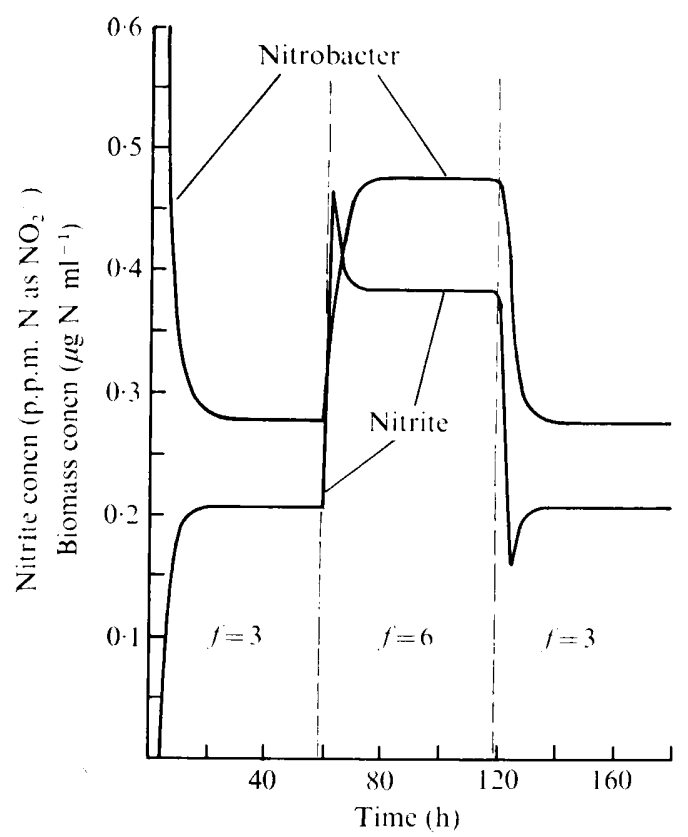

Fig. 1. Changes in biomass and nitrite concentration during increase and decrease in flow rate $\left(f\right.$, in $\left.\mathrm{ml} \mathrm{h}^{-1}\right)$ as predicted by the finite difference model for a column supplied with I p.p.m. $\mathrm{N}$ as $\mathrm{NO}_{2}^{-}$and inoculated with Nitrobacter $\left(\mu=2 \cdot 0 \mathrm{~h}^{-1}, K_{\mathrm{s}}=3.00\right.$ p.p.m. N as $\left.\mathrm{NO}_{2}^{-}, k_{1}=0.68 \mathrm{~h}^{-1}\right)$.

Thus growth is governed by Monod kinetics while conversion is governed by the zeroorder rate constant $k_{2}$. Assimilation is not considered so that at steady state:

$$
\bar{S}_{2}=S^{0}-\bar{S}_{1}
$$

where the bars indicate steady state concentrations. Steady state solution of equations I and 3 gives:

$$
\begin{gathered}
\quad \frac{k_{2} \bar{m}}{D}=S^{0}-\bar{S}_{1}+\frac{\mu \bar{S}_{1}}{K_{s}+\bar{S}_{1}} \cdot \frac{\bar{m}}{D} \\
\text { i.e. } \quad k_{2}=\frac{\mu \bar{S}_{1}}{K_{s}+\bar{S}_{1}}
\end{gathered}
$$

i.e. the zero-order rate constant equals the actual growth rate of Nitrobacter. From equation $\mathrm{I}$

$$
\bar{S}_{1}=S^{0}-\frac{\mu \bar{S}_{1} m}{K_{\mathrm{s}}+\bar{S}_{1}} \cdot \frac{\mathrm{I}}{D}
$$

Thus $\bar{S}_{1}$ and consequently actual growth rate will vary with dilution rate and $k_{1}$ cannot be considered as constant. The model was therefore modified by eliminating equation 3 and values of $S_{2}$ were calculated using the equation $S_{2}=S^{0}-S_{1}$ and equations $\mathrm{r}$ and 2 only were used. Thus, all the nitrate formed comes from conversion of nitrite for growth of Nitrobacter. Growth follows Monod kinetics but population size is determined by a logistic term, in effect giving a maximum population, the size of which is determined by the nitrite concentration.

\section{Predictions of the finite difference model}

Predictions of this model for high substrate concentration are not significantly different from previous models as all predict a constant maximum population will be established throughout the column so that distribution with depth need not be considered. At low 

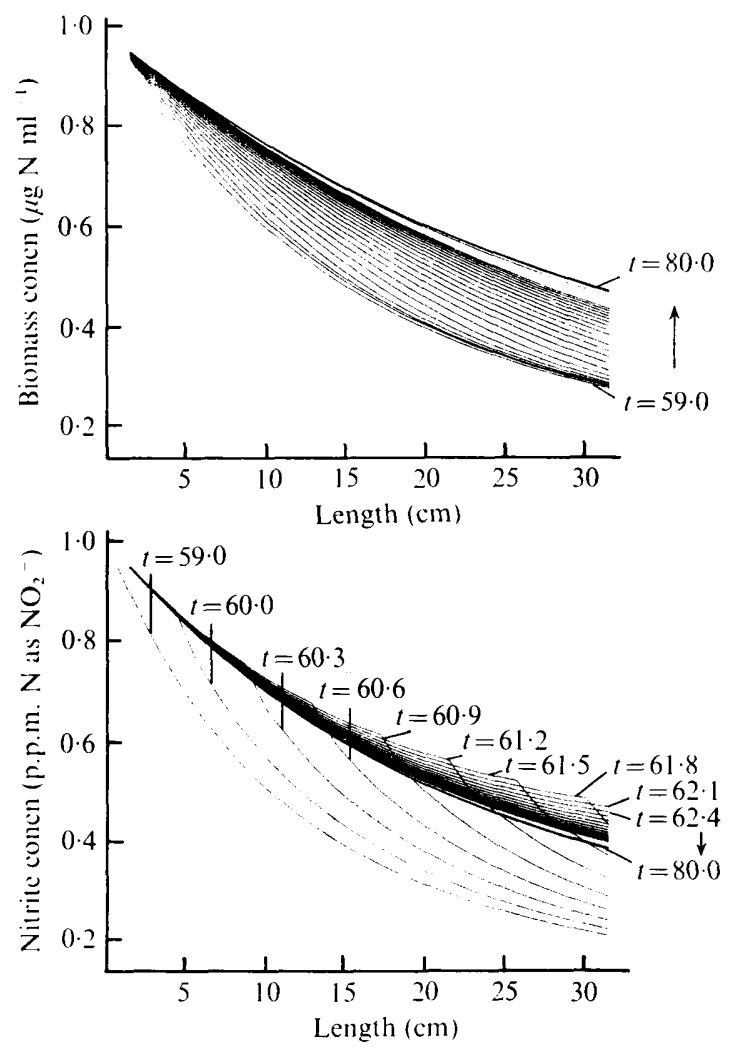

Fig. 2. Nitrite and biomass concentrations as functions of time $(t)$ and distance following the increase in flow rate at $60 \mathrm{~h}$.

substrate concentrations this is not the case and results of simulating this model, using parameter values from Bazin \& Saunders (1973) for increase and decrease in flow rate with a supply of I p.p.m. $\mathrm{N}$ as $\mathrm{NO}_{2}{ }^{-}$, show that numbers of Nitrobacter smoothly approach steady state values (Fig. I). Increasing the flow rate increases the nitrite concentration within the column and the Nitrobacter population therefore increases to a new steady state level. Nitrite concentration, however, rises to a peak and then falls to a new steady state level, higher than that at the previous flow rate. Conversely, decreasing the flow rate produces an undershoot in nitrite while the Nitrobacter population falls smoothly to its former level.

This overshoot phenomenon can be explained by changes in biomass. The steady state biomass at the lower flow rate, $f_{1}$, is less than that at the higher rate, $f_{2}$, due to higher nitrite concentration. When the flow rate is increased, biomass is less than the final level and the initial conversion rate is consequently lower. As biomass increases, the conversion rate increases and nitrite concentration then falls to its steady state level. The converse occurs when the flow rate is decreased.

As well as this qualitative difference, there are also quantitative differences caused by the presence of a biomass gradient down the column, due to variation in nitrite concentration. This gradient is illustrated in Fig. 2 during the increase in flow rate at $60 \mathrm{~h}$. The initial discontinuities correspond to movement of the front of higher nitrite concentration through the column and are followed by smooth progression to the new steady state value.

This overshoot prediction is unique to the model and the following experiments were designed to test this feature of the model. 


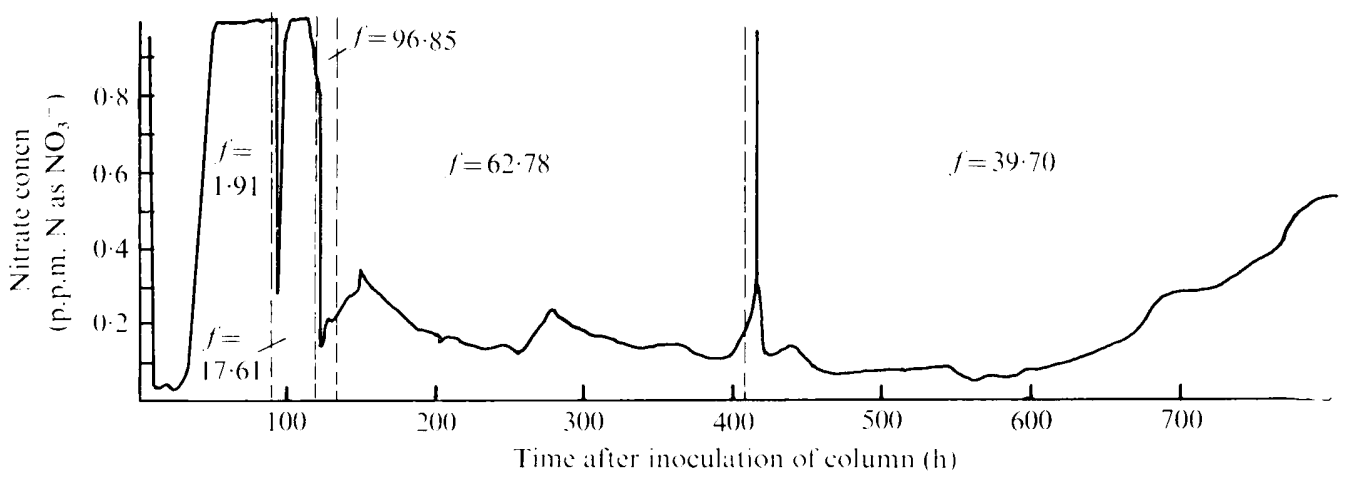

Fig. 3. Changes in nitrate concentration with time for a column inoculated with a $10^{-1}$ dilution of Nitrobacter. Line fitted through 99r points. Samples were taken at intervals of $60 \mathrm{~min}$ from o to II $5 \mathrm{~h}, 10 \mathrm{~min}$ from I 15 to $142 \mathrm{~h}, 50 \mathrm{~min}$ from $\mathrm{I} 42$ to $417 \mathrm{~h}, 40 \mathrm{~min}$ from 417 to $468 \mathrm{~h}, 60 \mathrm{~min}$ from 468 to $576 \mathrm{~h}$ and $90 \mathrm{~min}$ from 576 to $820 \mathrm{~h}$. Flow rate $(f)$ in $\mathrm{ml} \mathrm{h}^{-1}$.

\section{METHODS}

The experimental system consisted of a column of glass beads inoculated with Nitrobacter (Prosser \& Gray, 1977). The medium of Smith \& Hoare (1968) containing I p.p.m. N as $\mathrm{NaNO}_{2}$ was supplied at a constant flow rate which was varied in a stepwise fashion in each experiment. Four experiments were carried out with decreasing inoculum size. The inocula were dilutions of suspensions from batch cultures of Nitrobacter in Smith \& Hoare's medium with 50 p.p.m. $\mathrm{N}$ as $\mathrm{NO}_{2}{ }^{-}$containing between $10^{8}$ and $10^{9}$ bacteria $\mathrm{ml}^{-1}$. Excess suspension was drained through the columns, except after inoculation of a $10^{-6}$ dilution when it was poured off.

The model was simulated on a 1906 ICL digital computer. Differential equations were solved numerically using the fourth order Runge-Kutta approximation method (Fröberg, 1970) and results were plotted on a Calcomp 936 graph plotter.

\section{RESULTS}

\section{Inoculation with a $\mathrm{IO}^{-1}$ dilution of Nitrobacter}

Changes in nitrate with time at different flow rates are given in Fig. 3. After a lag of $35 \mathrm{~h}$ there was a linear increase in nitrate concentration to a value equal to that of the input nitrite concentration. The linear increase can be explained by simultaneous commencement of metabolic activity by bacteria throughout the column.

The flow rate was increased at $93 \mathrm{~h}$ but was immediately reduced to prevent excessive build up of pressure within the system. The net increase in flow rate, however, resulted in a sharp drop in nitrate, followed by a linear increase over approximately $6 \mathrm{~h}$ to a level corresponding to complete conversion. Six hours is much less than the generation time of Nitrobacter and the increase is therefore not due to growth. This undershoot was also much shorter than those predicted when the model was simulated using growth rate values measured for Nitrobacter. This can be explained by the presence of active bacteria and bacteria which are viable but inactive due to exhaustion of nitrite lower down the column. When nitrite reaches these bacteria after an increase in flow rate there is a short lag period before they regain full activity. Corresponding overshoots would not occur (and were not observed) after a decrease in flow rate as only active bacteria provide observable effects and the inactivation of bacteria would not be noticed. This effect is similar to that found at high substrate concentrations when oxygen is limiting (Prosser \& Gray, 1977).

It was possible to maintain a flow rate of $63 \mathrm{ml} \mathrm{h}^{-1}$ at which conversion was incomplete and a steady state nitrate concentration of 0.13 p.p.m. was obtained. Alternate samples were refrigerated before analysis and these had lower nitrate concentrations, indicating 


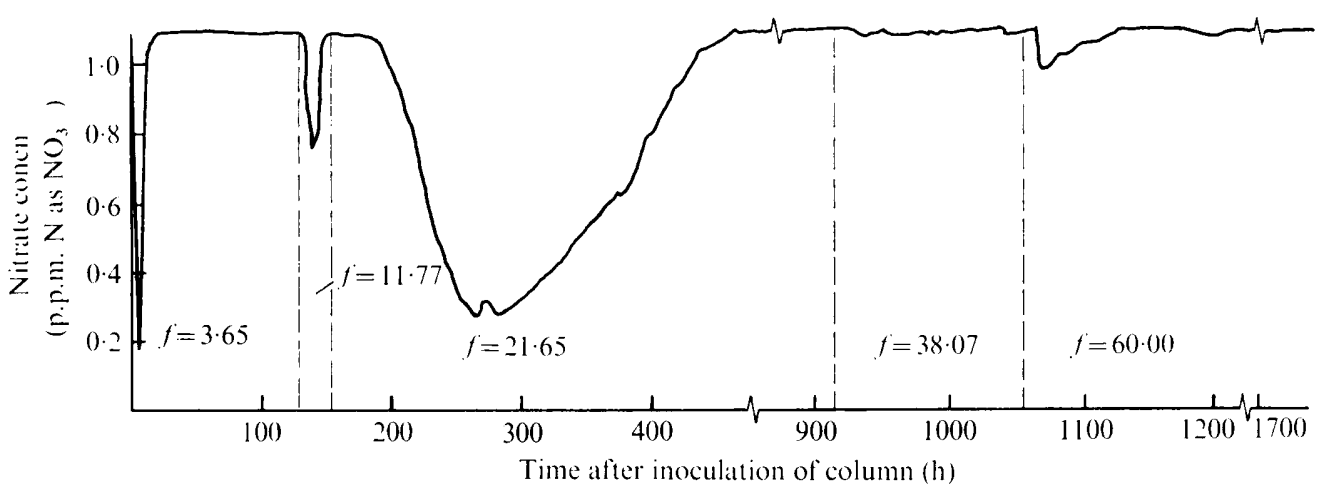

Fig. 4. Changes in nitrate concentration with time for a column inoculated with a $2.5 \times 10^{-3}$ dilution of Nitrobacter. Line fitted through 904 points. Samples were taken at intervals of $60 \mathrm{~min}$ from o to $\mathrm{I} 25 \mathrm{~h}, 90 \mathrm{~min}$ from $\mathrm{I} 25$ to $\mathrm{I} 34 \mathrm{~h}, 60 \mathrm{~min}$ from $\mathrm{I} 34$ to $822 \mathrm{~h}, 30 \mathrm{~min}$ from 822 to $858 \mathrm{~h}, 60 \mathrm{~min}$ from 858 to $898 \mathrm{~h}, 90 \mathrm{~min}$ from 898 to $1060 \mathrm{~h}$, ro min from I060 to II IO $\mathrm{h}, 60 \mathrm{~min}$ from I I IO to I6IO $\mathrm{h}$ and $90 \mathrm{~min}$ from $\mathrm{I} 6 \mathrm{IO}$ to $\mathrm{I} 750 \mathrm{~h}$. Flow rate $(f)$ in $\mathrm{ml} \mathrm{h}^{-1}$.

washout of cells, which is not considered by any of the models but which might be expected at the high flow rate used.

The overall conversion rate at the steady state was lower than that obtained in the system at 50 p.p.m. $\mathrm{N}$ as $\mathrm{NO}_{2}^{-}$(Prosser \& Gray, 1977) due to lower populations as a result of lower growth rates and increased washout.

These results show the necessity for a lower inoculum and lower dilution rates to prevent complete conversion within the column and consequent loss of information. They also highlight two important factors not considered by the model: the existence of active and non-active bacteria, and removal of bacteria by washout.

\section{Inoculation with a $\mathrm{IO}^{-2}$ dilution of Nitrobacter}

After inoculation with Nitrobacter, and at a flow rate of $2.63 \mathrm{ml} \mathrm{h}^{-1}$, there was a lag followed by a linear increase in nitrate concentration up to complete conversion. Increasing the flow rate to $23.05 \mathrm{ml} \mathrm{h}^{-1}$ at $320 \mathrm{~h}$ produced a sudden drop in nitrate concentration due only to change in flow. In the previous experiment this was followed by an increase in nitrate to a level corresponding to complete conversion of nitrite due to reactivation of bacteria previously not converting nitrite. This did not occur here possibly because the lower inoculum would result in exhaustion of nitrite nearer the base of the column and fewer or no inactive bacteria. There was a subsequent prolonged decrease in nitrate concentration until the end of the experiment at $420 \mathrm{~h}$. This can be explained by greater washout of bacteria at the higher flow rate. The results from the following experiment, however, suggest that the period 320 to $420 \mathrm{~h}$ was the first half of an undershoot similar to that predicted and that continuation of the experiment would have resulted in an increase in nitrate concentration.

\section{Inoculation with $2.5 \times 10^{-3}$ dilution of Nitrobacter}

Despite the reduced inoculum, complete conversion of nitrite still occurred (Fig. 4). An increase in flow rate at $\mathrm{I} 34 \mathrm{~h}$ resulted in a sharp drop in nitrate concentration followed by a rise to complete conversion as in the first experiment. A further increase in flow rate resulted in a steady decrease in nitrate concentration, similar to that in the previous experiment, down to 0.3 p.p.m. $\mathrm{N}$ as $\mathrm{NO}_{3}{ }^{-}$followed by an increase due to growth of Nitrobacter. This may correspond to the predicted undershoot.

The Nitrobacter population then reached such levels that two further increases in flow rate gave no measurable decreases in nitrate concentration and complete conversion 


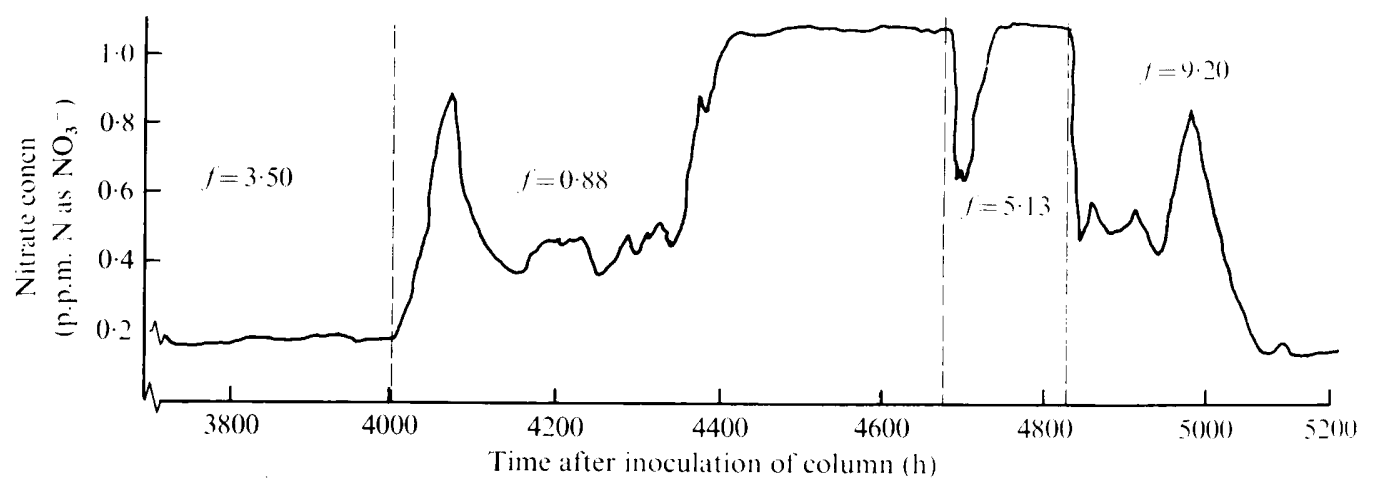

Fig. 5. Changes in nitrate concentration with time for a column inoculated with a $10^{-6}$ dilution of Nitrobacter. Line fitted through 1030 points. Samples were taken at intervals of $90 \mathrm{~min}$ from 3690 to $468 \mathrm{o} \mathrm{h}, 60 \mathrm{~min}$ from 4680 to $4730 \mathrm{~h}, 90 \mathrm{~min}$ from 4730 to $4855,60 \mathrm{~min}$ from 4855 to $4880 \mathrm{~h}$ and $90 \mathrm{~min}$ from 4880 to $5200 \mathrm{~h}$. Flow rate $(f)$ in $\mathrm{ml} \mathrm{h}^{-1}$.

occurred at a flow rate of $60 \mathrm{ml} \mathrm{h}^{-1}$. A similar flow rate and dilution rate in the first experiment did not give complete conversion but this column was run for longer allowing establishment of a larger Nitrobacter population. Any washout of bacteria, as in the previous experiment, did not reduce these populations below the level required for complete conversion.

\section{Inoculation with $a \mathrm{IO}^{-6}$ dilution of Nitrobacter}

Here three methods were used to avoid complete conversion of nitrite to nitrate. Firstly, a shorter column of beads $(80 \mathrm{~mm})$ was used so that dilution rates were greater than in other experiments for the same flow rates. Secondly, a smaller inoculum was used and, thirdly, the excess suspension was poured off and not drained through the column, to avoid increase in numbers due to filtration.

A steady state was obtained after approximately $3800 \mathrm{~h}$ with nitrate concentration equal to 0.183 p.p.m. (Fig. 5). Decreasing the flow rate resulted in an overshoot in nitrate with a maximum of 0.4 p.p.m. approximately $150 \mathrm{~h}$ after the change in flow. This overshoot was smaller than the undershoot in the previous experiment and the return to a steady state was quicker ( $150 \mathrm{~h}$ as opposed to $250 \mathrm{~h}$ ). In fact a new steady state may not have been established as there was then an increase in nitrate concentration due to growth of Nitrobacter until conversion was complete.

An increase in flow rate at $4690 \mathrm{~h}$ resulted in a decrease in nitrate concentration to 0.66 p.p.m. where it remained for about $20 \mathrm{~h}$ before increasing to give complete conversion, the rate of increase indicating growth of Nitrobacter rather than reactivation of bacteria.

After a further increase in flow rate, nitrate concentration dropped to about 0.55 p.p.m. but a blockage in the column caused variation in the flow rate resulting in the overshoot at $5000 \mathrm{~h}$. A steady state was eventually established with a nitrate concentration of o. I5 p.p.m. This is approximately equal to the steady state concentration at $3990 \mathrm{~h}$ despite a threefold increase in flow rate indicating a similar increase in biomass during this period.

\section{DISCUSSION}

The main qualitative difference in predictions between the model described above and previous models is the occurrence of undershoots and overshoots following changes in flow rate. Some experiments (the third and fourth) gave undershoots and overshoots in nitrate concentration lasting approximately $\mathrm{I} 50 \mathrm{~h}$ and $200 \mathrm{~h}$ following increase and decrease in flow rate respectively. Simulation of the model using values of $\mu, K_{\mathrm{s}}$ and $k_{1}$ measured for Nitrobacter in batch culture of $0.045 \mathrm{~h}^{-1}, 0.43$ p.p.m. $\mathrm{N}$ as $\mathrm{NO}_{2}{ }^{-}$and $0.33 \mathrm{~h}^{-1}$ gave under- 
shoots and overshoots of this length. However, these were asymmetrical consisting of a sharp rise or fall in nitrate concentration followed by a gradual decrease or increase to the new steady state value. Experimental undershoots and overshoots were symmetrical, and there is no definite evidence for re-establishment of new steady states after undershoots and overshoots. These discrepancies may be due to deficiencies in the description of growth and, in particular, growth limitation.

The logistic equation used here describes accurately limitation by space, but as conversion of nitrite was not complete at the end of the fourth experiment at a relatively low flow rate (Fig. 5), the maximum population may not have been reached. The presence of activity in effluent samples suggests that washout of bacteria is an important factor and an attempt was therefore made to incorporate this in the model. Bazin \& Saunders (1973) used three expressions for growth limitation:

$$
\begin{aligned}
& f_{1}=-k m^{2} \\
& f_{2}=-D m \\
& f_{3}=-D m^{2}
\end{aligned}
$$

where $k$ is a constant. The first of these gave the best fit to their experimental data and was used in the above model. The third gave poor fit but the second gave reasonable fit and was used to modify equation 2 to give:

$$
\frac{\mathrm{d} m}{\mathrm{~d} t}=\frac{\mu S_{1} m}{K_{\mathrm{s}}+S_{1}}-k_{1} D m
$$

Simulation of this model for a normal continuous culture system predicted smooth approaches to new steady states after changes in flow rate but incorporation into the finite difference model produced overshoots and undershoots. However, although the time taken to reach new steady states was comparable with that observed, the initial increase or decrease in nitrate concentration was much quicker than that observed, suggesting that growth and washout are more important factors than flow rate under these conditions and that both of these were described in too simple a fashion.

For example, washout may be more complicated than the simple representation above as some bacteria may be more susceptible to washout than others, the process may be growth rate dependent and washout would then be balanced by growth of bacteria remaining to replace those lost. Also bacteria may not adjust immediately to new growth rates at different nitrite concentrations. The minimum of an undershoot may therefore represent adjustment to the new flow rate, followed by increase in nitrate concentration due to faster growth of Nitrobacter.

The model provides an insight into possible mechanisms for dynamics of growth in column systems and provides a partial explanation for observed behaviour. Experiments at low nitrite concentrations exposed deficiencies in this and other models in their descriptions of growth rate and growth limitation. The model for cell growth of Williams (1967) may be useful as this predicts lag phases after changes in environmental conditions.

Experimental testing of the model may be facilitated by use of larger beads (reducing surface area and bacterial numbers) and by enumeration of the bacteria washed out. Results also confirmed the importance of determining numbers of nitrifiers when considering nitrification and models could be tested more critically if sampling and enumeration of bacteria at different depths could be achieved. The system of Ardakani, Rehbock \& McLaren (1973) with sampling ports along the column may be useful but any sampling process will disturb the system to some extent.

J. I. Prosser wishes to acknowledge receipt of a N.E.R.C. Research Studentship. 


\section{REFERENCES}

ARdakani, M. S., REHBOCK, J. T. \& MCLAREN, A. D. (1973). Oxidation of nitrite to nitrate in a soil column. Proceedings of the Soil Science Society of America 37, 53-56.

Bazin, M. J. \& Saunders, P. T. (1973). Dynamics of nitrification in a continuous flow system. Soil Biology and Biochemistry 5, 53 I-543.

FröBERG, C. E. (1970). Introduction to Numerical Analysis. London: Addison-Wesley.

LeEs, H. \& Quastel, J. H. (1946). Biochemistry of nitrification in soil. 2. The site of soil nitrification. Biochemical Journal 40, 815-823.

Prosser, J. I. \& Gray, T. R. G. (I977). Nitrification studies at non-limiting substrate concentrations. Journal of General Microbiology 102, I I 1-1 17.

Saunders, P. T. \& BAZIN, M. J. (I973). Nonsteady state studies of nitrification in soil: theoretical considerations. Soil Biology and Biochemistry 5, 545-557.

Smith, A. J. \& HoAre, D. S. (I968). Acetate assimilation by Nitrobacter agilis in relation to its 'obligate autotrophy'. Journal of Bacteriology 95, 844-855.

Williams, F. M. (1967). A model of cell growth dynamics. Journal of Theoretical Biology $\mathbf{5}$, 190-207. 